\title{
Lumbar Spinal Arthroplasty: Clinical Experience
}

\author{
Fred H. Geisler \\ Chicago Back Institute, Swedish Covenant Hospital, N. Francisco, Chicago, IL
}

USA

\section{Introduction}

Lumbar spinal arthroplasty was first reported in clinical settings more than 10 years ago by Griffith et al ${ }^{1}$. This early experience was acquired with the first lumbar artificial disc, the CHARITÉ I, in patients with degenerative disc disease. Since that time, a randomized controlled trial comparing arthroplasty with the CHARITÉ Artificial Disc vs. anterior lumbar interbody fusion with the BAK Cage and iliac crest bone was completed. Multiple other lumbar arthroplasty devices have been developed subsequent to the CHARITÉ and are undergoing or completing clinical trials.

Unlike other spinal medical devices, lumbar discs are required by the Food and Drug Administration to complete randomized controlled trials (RCT) prior to market approval in the United States. As a result, lumbar arthroplasty devices have undergone more scrutiny and clinical evaluation than any other spinal medical devices. Specifically, a new device, the ProDisc-L, was granted FDA approval in 2006 and was described in a recent peer-review publication². In addition, the Maverick Total Disc Arthroplasty System (Medtronic Sofamor Danek), Kineflex Lumbar Disc (SpinalMotion), and FlexiCore Intervertebral Disc (SpineCore/Stryker) lumbar discs have both completed their randomized enrollments and are currently in continued access (non-randomized) mode.

All these ongoing and completed randomized clinical trials have generated a large body of evidence on the safety and efficacy of arthroplasty for lumbar spine in clinical applications and, in many cases, in Level-1 publications.

The safety and efficacy of arthroplasty are not the only parameters discussed in the $>60$ clinical papers published over the last 6 years. In fact, significant insights were developed in the impact of arthroplasty on sagittal alignment and motion, possible adverse events and reoperation, as well as optimal patient selection and indication. Surgical technique and health economics papers have also been generated in an effort to fully understand the clinical and societal impact of this new technology. This review paper is aimed at providing an overview of all the existing clinical data related to spinal arthroplasty.

\section{Materials and methods}

A search was conducted on the OVID and COCHRANE Library database to collect all clinical data relevant to spinal arthroplasty. Specifically, the following keywords were used: 
(CHARITÉ Artificial Disc or ProDisc-L or Maverick Total Disc Arthroplasty System or KineFlex or FlexiCore Intervertebral Disc) and (disc) and (lumbar). The search was limited to English-language papers. Preclinical, biomechanical, and review papers were excluded from the final paper selection. In addition, papers describing obsolete devices (CHARITÉ I and CHARITÉ II) were also excluded from the study. A total of 60 papers were analyzed herein and subdivided by key topic, as following: 1) General clinical outcomes; 2) Radiographic Analysis: Range of motion, heterotopic ossification and sagittal balance analyses; 3) facet and adjacent-level degeneration; 4) Revisions and revision strategies; 5) Surgical technique; 6) Complications; 7) Special patient population analyses; and 8) Health economics evaluations.

\section{Results}

\subsection{General clinical outcome results}

General clinical outcome results were available for the CHARITÉ Artificial Disc, the ProDisc-L, the Maverick Total Disc Arthroplasty System, and the FlexiCore Intervertebral Disc. However, level-1 data was only available for the CHARITÉ Artificial disc and the ProDisc-L, as the final study FDA IDE results for the Maverick Total Disc Arthroplasty System and the FlexiCore Intervertebral Disc have yet to be published.

The CHARITÉ Artificial Disc manuscripts described clinical outcomes as early as 1 year 3 and up to 13 years post-operative ${ }^{4}$. The short/medium term papers included herein typically disclosed early analyses from single sites involved in the CHARITÉ Artificial Disc IDE study comparing arthroplasty with CHARITÉ Artificial Disc vs. anterior interbody fusion with BAK cage and autograft 3,5,6. The complete RCT at 2-year follow-up included 205 arthroplasty and 99 fusion patients, and was thoroughly described in two manuscripts, one focused on clinical outcomes ${ }^{7}$, the other on radiographic outcomes ${ }^{8}$. Three additional medium- and long-term studies were also found: 2 papers with 10-year follow-up 4,9 and one with an average of 6.6 year follow-up ${ }^{10}$.

Safety and efficacy of arthroplasty was demonstrated in all short- and medium-term studies. Specifically, at 2-years post-operative, Blumenthal and McAfee reported no device-related complications and a reoperation rate of $5.4 \%$ (vs. 9.1\% in the control arm). Efficacy was also demonstrated using validated disability (ODI) and pain (VAS) clinical outcomes tools. At 2 years, the reduction in ODI reached $48.5 \%$ (vs. $42.4 \%$ in the control group) and the absolute reduction in VAS reached 40.6 points (vs. 34.1 points in the control group) 7,8 .

Two of the three long-term studies confirmed these findings. Lemaire et al reported 10year follow-up results in 100 patients. ${ }^{4}$ This study included 54 patients operated at one level, 45 patients operated at two levels and one patient operated at 3 levels. Overall, authors reported excellent or good clinical outcomes in $90 \%$ cases. In a second long-term study, David et al presented 10-year data on 106 patients 9 . Only one-level surgeries were performed in this study. Excellent or good clinical outcome was obtained in $82.1 \%$ patients. Both papers thus concluded that arthroplasty was a viable option for disc degeneration.

Recently, a third, medium-term paper was published by Ross et al, describing the longterm effect of arthroplasty in 160 patients (226 CHARITÉ Artificial Discs). This paper reported a cumulative survival rate at 156 months of $35 \%$ and a mean ODI score 
improvement of $14 \%$. Implant removal was also described in 12 patients ${ }^{10}$. These relatively poor findings were further discussed in two Letters to the Editors, which pointed out mathematical inconsistencies and overall clinical flaws in the manuscript, and further highlighted the need of proper surgical technique and patient selection for optimal clinical outcome 11, 12 .

The ProDisc-L manuscripts described clinical outcomes as early as 3 months ${ }^{13}$ and up to 8.7 years post-operative ${ }^{14}$. As described above for CHARITÉ Artificial Disc, The shortterm papers typically disclosed early findings from one or two of the sites that participated in the randomized controlled trial comparing ProDisc-L against a 360 degree fusion $13,15-21$. The complete RCT at 2-year follow-up included 161 arthroplasty and 75 fusion patients ${ }^{2}$. The long-term data included 64 patients operated at one site, of which 55 were available between 7 and 11 years post-operative, for clinical and radiographic follow-up ${ }^{14}$. All these studies concluded similarly that disc arthroplasty, at all evaluated time points, was safe and resulted in complication and/or re-operation rates comparable to those generally accepted for spinal surgery (complication rate of $9 \%$ at 8.7 years 14 ; there were no major complications, but a reoperation rate of $3.7 \%$ at 2 years was reported 2 ). In addition to safety, efficacy of spinal arthroplasty was also shown herein as all cases presented significant improvements in pain and disability. The final RCT data reported improvements in the arthroplasty group in Visual Analogue Scores (VAS) for pain by an average $39-\mathrm{mm}$, and in disability, as determined by the Oswestry Disability Index (ODI), by 28 points. It is worth noting, however, that the ODI tool utilized in this RCT was not the validated and widely accepted ODI methodology Version 1.0 as defined by Fairbanks et $\mathrm{al}^{22}$. In a Letter to the Editor, Fairbanks denounced the use of the so-called Oswestry Disability Index in the ProDisc-L study and thus cast doubt on the validity of the disability improvement outcomes observed herein ${ }^{23}$.

The 2 clinical data publications on the MAVERICK device were both based on the same data set of 64 patients, collected at one site 24,25 . The clinical outcomes were described using the ODI Version 1.0 and VAS scores. The efficacy of arthroplasty was once again demonstrated using these tools, as ODI scores decreased by an average of 20.7 points and VAS scores by 4.4 points. As for the FlexiCore Intervertebral Disc, only one paper was recently published ${ }^{26}$. This manuscript describes the clinical outcomes of 44 patients, of which only 6 were available for 2-year follow-up. While the clinical relevance of this data may therefore be questionable, authors still concluded that the device may be safe and efficacious but that the data was not representative of the entire patient cohort.

\subsection{Radiographic analyses: Range of motion, heterotopic ossification and sagittal balance analyses}

Radiographic evaluations such as ROM, heterotopic ossification, and sagittal balance have been broadly analyzed for the CHARITÉ Artificial Disc, the ProDisc-L, as well as the Maverick Total Disc Arthroplasty System.

Unlike other clinical and radiographic outcomes, accurate measurement of ROM was shown to be challenging and, to some extent, subjective, as patient positioning, imaging staff training, and other factors unrelated to the actual motion potential of the spine were shown to impact final readings 27 . Using ProDisc-L cases, Lim et al evaluated different methodologies and associated error margins for the measurement of ROM from 
radiographic images. Specifically, Lim et al concluded that a ROM of at least 4.6 degs must be observed in order to be $95 \%$ certain that a given device had any sagittal motion at all. Similarly, changes needed to be at least 9.6 degs in ROM in order to confirm at $95 \%$ that change in motion really happened ${ }^{28,}{ }^{29}$. These technical limitations might explain the inconsistent ROM data, particularly for ProDisc-L cases, found in the published literature. The flexion-extension ROM results from the 2-year RCT were determined at 7.7 degrees and 4.67 degrees and characterized as a normal ROM 2. However, at 8.7 years post-operative, Huang et al reported a ROM less than that reported in asymptomatic normal individuals, with an average motion of 3.8 degs ${ }^{30}$. In a 2006 prospective study on 41 patients with 2 year follow-up, Leivseth et al also reported that the device fails to restore normal segmental motion, while another retrospective study on 26 patients concluded that sagittal balance and ROM significantly improved after lumbar arthroplasty 31 .

Less controversy was observed when reviewing ROM data for the CHARITÉ Artificial Disc. A complete manuscript was dedicated to the radiographic data of the 2-year RCT of the CHARITÉ Artificial Disc ${ }^{8}$. In this study, arthroplasty patients had a $13.6 \%$ increase in motion from preoperative to the 2-year post-operative time point. The ROM also correlated to device placement, as poor device placement resulted in a statistically significant reduction in motion. At 10-year follow-up, David reported an average 10.1 degs ROM, a value very similar to the 10.3 degs reported by Lemaire et al in their 10-year follow-up study ${ }^{4,9}$.

Le Huec et al published the only data available on radiographic findings after arthroplasty with the Maverick Total Disc Arthroplasty System. In their study, Le Huec et al broadened their analysis to include sagittal alignment and pelvic tilt ${ }^{24}, 32$. Using data related to 35 patients at an average 14 months post-operative, authors showed maintenance of overall lordosis and unchanged sacral and pelvic tilts, following arthroplasty.

More recently, a study by Tournier comparing all three - CHARITÉ Artificial Disc, ProDisc-L and Maverick Total Disc Arthroplasty System - further refined the analyses from LeHuec on pelvic and sagittal tilt. In this study, authors found no difference in ROM between prostheses and observed maintenance of sagittal balance before and after surgery with all devices. However, modifications of the lumbar curvature were observed ${ }^{33}$.

The issue of heterotopic ossification in clinical cases of lumbar arthroplasty has been presented by McAfee et al and, more recently, by Tortolani et al ${ }^{34}, 35$. In his 2003 paper, McAfee introduced a novel method to characterize spinal heterotopic ossification. This methodology was applied by Tortolani et al in reviewing the 276 arthroplasty patients from the CHARITÉ Artificial Disc RCT (randomized and non-randomized cases). From this analysis, $4.3 \%$ cases of heterotopic ossification were noted. However, heterotopic ossification was not related to range of motion, as the authors concluded that no difference in the range of motionat 24-months post-operatively was found between the patients who had and those who did not have heterotopic ossification.

\subsection{Facets and adjacent-level degeneration}

Facet degeneration is currently a contraindication for arthroplasty. However, a few publications have investigated the impact of arthroplasty on index-level facet joints, as 
well as adjacent-level discs, in order to determine whether the added motion at the index level could slow down the natural progression of the disease at the facets and the adjacent-level joints.

Three long-term analyses evaluated adjacent-level degeneration, one with ProDisc-L at 8.7 years, and the other two with the CHARITÉ Artificial Disc at 10 years follow-up. In the ProDisc-L study, 24\% cases developed adjacent level degeneration by the latest follow-up time point. A correlation was also found between a low range of motion and the prevalence of adjacent level degeneration: all patients with adjacent-level degeneration had a ROM 1 ess than 5 degrees, while only 59\% of patients without adjacent-level degeneration had a ROM less than 5 degrees ${ }^{36}$. Lemaire et al and David reported $2(2 \%)$ and $3(2.8 \%)$ cases of adjacent-level degeneration at the latest time point, respectively ${ }^{4}, 9$. Lemaire et al and David also disclosed 11 cases $(11 \%)$ and 5 cases $(4.7 \%)$ with facet arthrosis at the latest time point, respectively.

The issue of facet degeneration was also recently discussed in a short-term study. From a 13patient case series with 12 months follow-up, Trouillier et al alluded to possible maintenance of facet joint integrity following arthroplasty with CHARITÉ Artificial Disc based on the favorable results from their series ${ }^{37}$. At the other end of the spectrum, Shim et al, at the 3-year time point, observed $36.4 \%$ and $32.0 \%$ increase in index-level facet degeneration and $19.4 \%$ and $28.6 \%$ adjacent level disc degeneration with the CHARITÉ Artificial Disc and the ProDisc-L, respectively.

\subsection{Revision and revision strategies}

The issue of possible revisability of arthroplasty devices represented a key concern when the first artificial disc, the CHARITÉ Artificial Disc, was introduced to the market. As such, multiple papers have focused on this issue and provided surgical and clinical insights to ensure appropriate approaches to revision surgery.

The first description of appropriate revision for a CHARITÉ Artificial Disc was presented by David ${ }^{38}$. In this single-case example, a CHARITÉ Artificial Disc was replaced at 9.5 years post-operative with another CHARITÉ Artificial Disc. The author concluded that revision of the disc with another disc could be safely and adequately performed and was thus an alternative to a revision fusion procedure. David also noted that, due to the inherent difficulty of an anterior approach, only experienced surgeons should undertake this operation. Further revision and explantation of the disc were also described by McAfee et al and Leary et al 39, 40. McAfee et al confirmed Davids experience and concluded that arthroplasty with the CHARITÉ Artificial Disc did not preclude any further procedures at the index level during primary insertion, with nearly one third being revisable to a new motion-preserving prosthesis and just over two thirds being successfully converted to ALIF and/or posterior pedicle screw arthrodesis, the original alternative procedure. Leary further implied that technical errors in position and sizing of the implant were largely to blame for further revision surgery. Finally, Punt et al reviewed 75 revision cases from the Dutch experience (estimated by the authors at approximately more than 1000 Dutch patients). In this series, patients were fused posteriorly either with removal of the disc or without removal of the disc. No statistically significant difference was observed between these 2 groups ${ }^{41}$. This paper included patients previously described by Van Ooij et $\mathrm{al}^{42}$. 


\subsection{Surgical technique}

The appropriate surgical technique with the CHARITÉ Artificial Disc as well as the ProDisc$\mathrm{L}$ was presented in 2 separate publications. Geisler et al provided a detailed account of the surgical technique for the CHARITÉ Artificial Disc, and dedicated an entire section of the paper to patient selection and preoperative planning, two critical aspects of successful spinal arthroplasty ${ }^{43}$. Authors also strongly recommended the availability of a spinal access surgeon to perform the approach, especially in revision cases. Finally, appropriate midline identification and positioning of the device also represented a critical discussion point in this paper. For the ProDisc-L, Gumbs et al retrospectively reviewed 64 cases of open retroperitoneal exposures and concluded that the approach was safe and, as discussed by Geisler et al, required a multidisciplinary team, such as an orthopedic and an access or general surgeon, to minimize complication rates ${ }^{44}$.

\subsection{Complications}

Complications from spinal arthroplasty have also been reported for all three devices. Most complications requiring revision surgery were resolved by either fusion and/or disc replacement surgery. Interestingly, the causes of these complications seemed to be devicespecific (i.e.; due to the design and/or make of the device).

For the ProDisc-L, the major complication described in the literature referred to the vertical split fracture of the vertebral body following total disc replacement. This occurrence was described by Shim et al in 2 separate cases that were not revised or treated surgically, but experienced prolonged back pain as a result ${ }^{45}$. An additional complication in the form of acquired spondylolysis was described by Schulte et $\mathrm{al}^{46}$. Authors attributed this complication to inaccurate implant size and positioning.

For the CHARITÉ Artificial Disc, the key complications were observed on the earlier devices, which were gamma sterilized in air and thus had a potential for oxidation of the core polyethylene nucleus ${ }^{38}$. Complications due to this oxidation process were described by Van Ooij et al (and Punt et al, as this paper reiterate data from the Van Ooij patient population) ${ }^{41}, 42$. This issue was resolved with a process change in 1998 to gamma sterilization in nitrogen. In a review of the RCT patient population, Geisler et al also evaluated the rate of neurological complications in the arthroplasty group vs. fusion group. The rate of neurological complication was described as exceedingly low in both groups with no statistically significant differences between groups.

While little has been published so far on the Maverick Total Disc Arthroplasty System, one article described an early removal of the Maverick Total Disc Arthroplasty System ${ }^{47}$. This removal was performed one year after implantation due to severe persistent back pain. Intraoperatively, gross metallosis around the articulation of the device was observed. The revision was successful and included a 360 degs fusion. Metallosis was thus cited as a potential complication for devices consisting of a metal-on-metal design. Zeh et al presented an additional potential complication: due to this metal-on-metal structure of the Maverick Total Disc Arthroplasty System, cobalt and chromium ions from the device were being released into the bloodstream ${ }^{48}$. In this study, cobalt and chromium ions from subjects implanted with the Maverick Total Disc Arthroplasty System were evaluated and compared to ion levels in total hip arthroplasty (THA) patients. Zeh et al found that concentrations of $\mathrm{Cr} / \mathrm{Co}$ measured in the serum were similar in terms of their level to the values measured in THA metal-on-metal combinations or exceed those values reported in the literature. As a 
result, while Zeh et al did not recommend holding back with the implantation of the device, they did suggest long-term clinical evaluations to determine the clinical impact of high ion levels in serum and also recommended discussions with patients on the potential health effects of the prosthesis.

\subsection{Special patient population analyses}

The clinical outcomes for selective patient populations (e.g.; smokers, $>60 \mathrm{yr}$ old) were discussed in multiple papers, more specifically for the ProDisc-L device. Bertagnoli et al lead these efforts with 4 publications presenting clinical data on arthroplasty with ProDisc-L in patients with: 1) single-level arthroplasty15; 2) multi-level arthroplasty16; 3) patients 60years or older ${ }^{49}$; 4) smokers 50 ; and 5) arthroplasty cases adjacent to a fused level 51 . While Bertagnoli et al repeatedly stated the importance of proper patient selection in each and every paper, all the results presented in these studies concluded that spinal arthroplasty with ProDisc-L successfully addressed low-back pain in these specific patient populations. Hannibal et al recently compared one- vs. two-level arthroplasty cases at the 2-year followup to try and establish whether one-level cases were experiencing greater clinical improvements as compared to the two-level cases. This hypothesis was not verified as differences in clinical improvements between one- and two-level cases were marginal 52 . Yaszay et al approached the problem from a different angle and evaluated patients outcomes based on a radiographic observation, i.e.; preoperative disc height 53 . Yaszay et al observed that patients with greater disc collapse experienced a greater benefit from total disc replacement, as compared to patients with less collapsed intervertebral discs. On average, patients in all of Bertagnoli et al and Yaszay et al series showed significant clinical improvement following arthroplasty.

Using the CHARITÉ Artificial Disc IDE RCT patient population, sub-analyses by patient types were also published by Guyer et al and Geisler et al. Specifically, patients were stratified by age at surgery (18-45 vs. 46-60) or whether they had had prior surgery or not ${ }^{54}$, 55. In both cases, there was no difference in clinical outcome between groups, whether patients were $18-45$ or $46-60$, or whether patient did or did not have prior surgery. Along the same trend, Geisler et al also evaluated the clinical outcomes of those patients from the CHARITÉ Artificial Disc RCT that did not improve with arthroplasty and needed revision surgery to a fusion. These patients (7.1\% of all arthroplasty cases) did not improve, despite the revision surgery, further highlighting the importance of proper patient selection, and possibly, the fact that patient selection still remains a somewhat approximate science $(59)^{56}$.

\subsection{Health economics evaluations}

The impact of spinal arthroplasty on health care economics were reviewed for both the CHARITÉ Artificial Disc and the ProDisc-L. Guyer et al analyzed the costs related to a CHARITÉ Artificial Disc arthroplasty compared to: 1) an anterior fusion with autograft: 2) anterior fusion with rhBMP-2 (Infuse Bone Graft and LT-Cages) as well as 3) instrumented posterior lumbar interbody fusion. This analysis included the cost of revision surgery at the rate estimated in the published literature. Guyer et al concluded that all fusion procedures were more costly than the arthroscopy approach by $12.0 \%$ (ALIF with autograft) to $36.5 \%$ (ALIF with rhBMP-2 and posterior fusion) $)^{57}$. 
A similar analysis by Levin et al evaluated the costs of 1- and 2-level arthroplasty vs. 360 degs fusion. This study did not include possible needs for revisions. Nevertheless, one-level arthroplasty cases were found to be less costly than one-level fusions $(\$ 35,592$ for arthroplasty vs. $\$ 46,280$ for 360 deg fusion) while two-level cases were similar for both groups $(\$ 55,524 \text { for arthroplasty vs. } \$ 56,823 \text { for fusion })^{58}$.

\section{Discussion}

From 2002 to 2008, a significant volume of data was made available on the clinical impact of arthroplasty. Sixty studies related to the clinical use of arthroplasty were published in peerreviewed papers, of which 35 described data collected prospectively and 18 represented data from multi-center studies. The total number of patients described in the literature for spinal arthroplasty is difficult to evaluate, since many studies are early data releases or subanalyses of the main randomized controlled trials performed for each new device. Thus, a given patient population might have been discussed in multiple papers. Overall, however, it was estimated that approximately 1,600 patients were included in the current literature.

The RCTs for the ProDisc-L and the CHARITÉ Artificial Disc both demonstrated the noninferiority of the arthroplasty procedure, compared to their respective controls (ALIF for CHARITÉ Artificial Disc, 360 degs fusion for ProDisc-L. The complete RCT data for Maverick Total Disc Arthroplasty System and FlexiCore Intervertebral Disc are not yet published.) For some specific clinical outcomes such as pain, disability, and hospital stay, arthroplasty patients experienced greater clinical outcomes at some of the follow-up time points. For the CHARITÉ Artificial Disc RCT, arthroplasty patients fared statistically better than fusion on pain and disability for all but the 24-month follow-up time point. Hospital stay was also significantly shorter in the CHARITÉ Artificial Disc group as compared to the fusion group. As for ProDisc-L, pain scores were statistically better in the investigational cohort as compared to control. In addition, most other case series, for any of the given arthroplasty products, including those with short- and long-term follow-up data, presented favorable overall outcomes for spinal lumbar arthroplasty.

The issues of range of motion, heterotopic ossification, and sagittal balance have also drawn significant attention. While accurate and reproducible measurement of the lumbar ROM may be a significant limitation to collect meaningful data, average ROM at the 2-year time point throughout the studies evaluated herein were at $\sim 7-10$ degs. In addition, restoration of sagittal balance was observed for each device, the CHARITÉ Artificial Disc, the ProDisc-L as well as the Maverick Total Disc Arthroplasty System.

This review contained however somewhat contradictory data on the issue of facet and adjacent-level degeneration. While two of the 10year studies showed very small instances of facet and/or adjacent-level degeneration, others reported nearly a $1 / 3$ of all cases with either CHARITÉ Artificial Disc or ProDisc-L developing changes in facet morphology. The inconsistency in these results points at potential surgeon-specific techniques and approaches that may impact the long-term benefits of both procedures.

Surgeon-specific variability in technique and proficiency was also cited in cases of revision. In fact, a study by Regan et al evaluating the occurrence of revision in low-volume vs. highvolume center, confirmed that surgeon in low-volume centers may incur greater perioperative complications, however none that affected long-term outcomes ${ }^{59}$. Nevertheless, 
revisions were often found to be associated with technical errors, such as errors in positioning or sizing of the implant.

This critical importance of proper technique was in fact described in the 2 technique papers discussed herein. Both these publications stressed the importance of proper patient selection, a recurrent theme in almost all arthroplasty discussions.

Three types of major complications were described or foreseen in the current literature: 1) vertebral body split due to the ProDisc-L keel design; 2) oxidation of the core polymer nucleus, a problem specific to first generation CHARITÉ I prosthesis; and 3) metallosis and long-term impact of metal ions in the body. While the issue of metallosis and metal ions still needs to be thoroughly investigated, the problems related to vertebral body split only occurred once in the published literature, and as such, may represent a rare occurrence, and that of core oxidation, has been since resolved with new sterilization techniques following which core oxidation of the polymer nucleus is not observed. Thus, possible complications related to devices with metal-on-poly designs seem to be fairly limited.

Finally, low rates of complication and adverse event were observed for most of all analyzed patients. For the ProDisc-L, smokers as well as patients 60 years of age seemed to experience similar benefit from the procedure. In all these studies, however, authors reiterated the importance of proper patient selection, thus concluding that, while all analyzed patient types experienced clinical benefit from the procedure, specific care must be given to only operate on appropriate patients.

Finally, no technology is sustainable in today's market place if its cost is prohibitive. Thus, the impact of arthroplasty on health economics was also investigated. Both, the CHARITÉ Artificial Disc and the ProDisc-L study came up at a lower cost than their fusion controls, whether potential revision costs were included or not. No data on cost exists yet for the Maverick Total Disc Arthroplasty System and the FlexiCore Intervertebral Disc.

\section{TDR surgery}

The typical diseased lumbar segment considered for artificial disc technologies at L4-L5 or L5-S1 has advanced degenerative disc disease with loss of vertical height and lordosis, dehydration changes, adjacent Modic endplate changes, and little motion on dynamic studies (see Figure 1). The natural progression of degenerative disease disc limits the joint mobility and this biomechanical fact places more forces on the adjacent levels then in the normal situation. The artificial lumbar disc, by restoring normal motion, height, and lordosis, will decrease the forces on the adjacent levels. Thus, theoretically, levels adjacent to a dynamically stabilized level may have beneficial effects compared to the natural history of the unoperated degenerative state. Clearly some patients will benefit from the decreased force on the adjacent vertebral level(s) following a dynamic stabilization (arthroplasty) compared to a static stabilization (fusion). Estimates of the rate and groups of patients at maximum benefit will need to await long-term clinical follow up studies with lumbar arthroplasty devices similar to the hip and knee arthroplasty registries. Also, although a dynamically stabilized level can be converted to a fusion, a fused level cannot be converted to a dynamic joint. Thus, artificial technology can be thought of as a definitive procedure for the vast majority of patients that can be converted to a fusion if the pain and functional goals are not met or degenerative changes occur in the posterior elements and the arthrodesis level is believed to be the pain generator. 


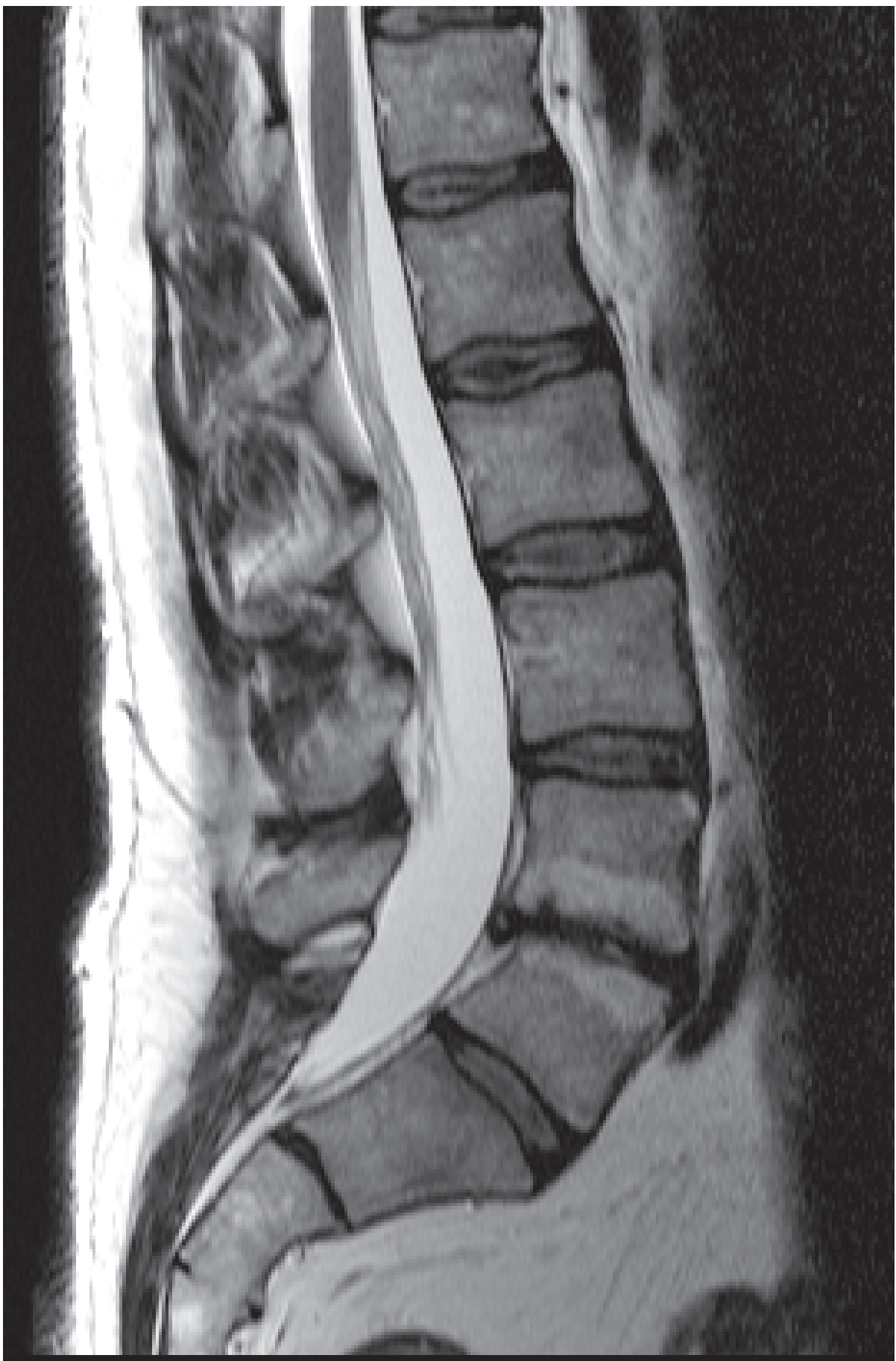




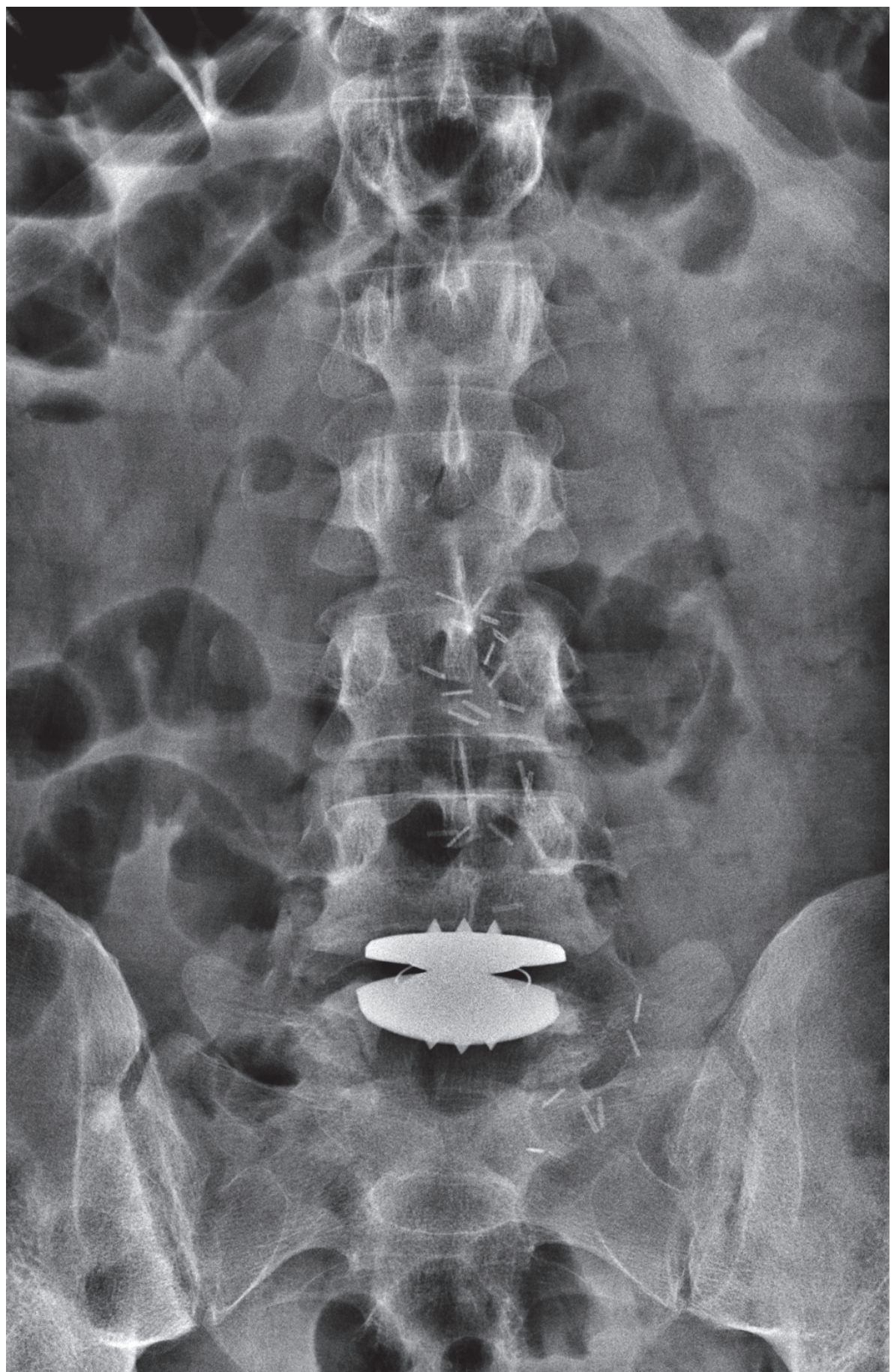




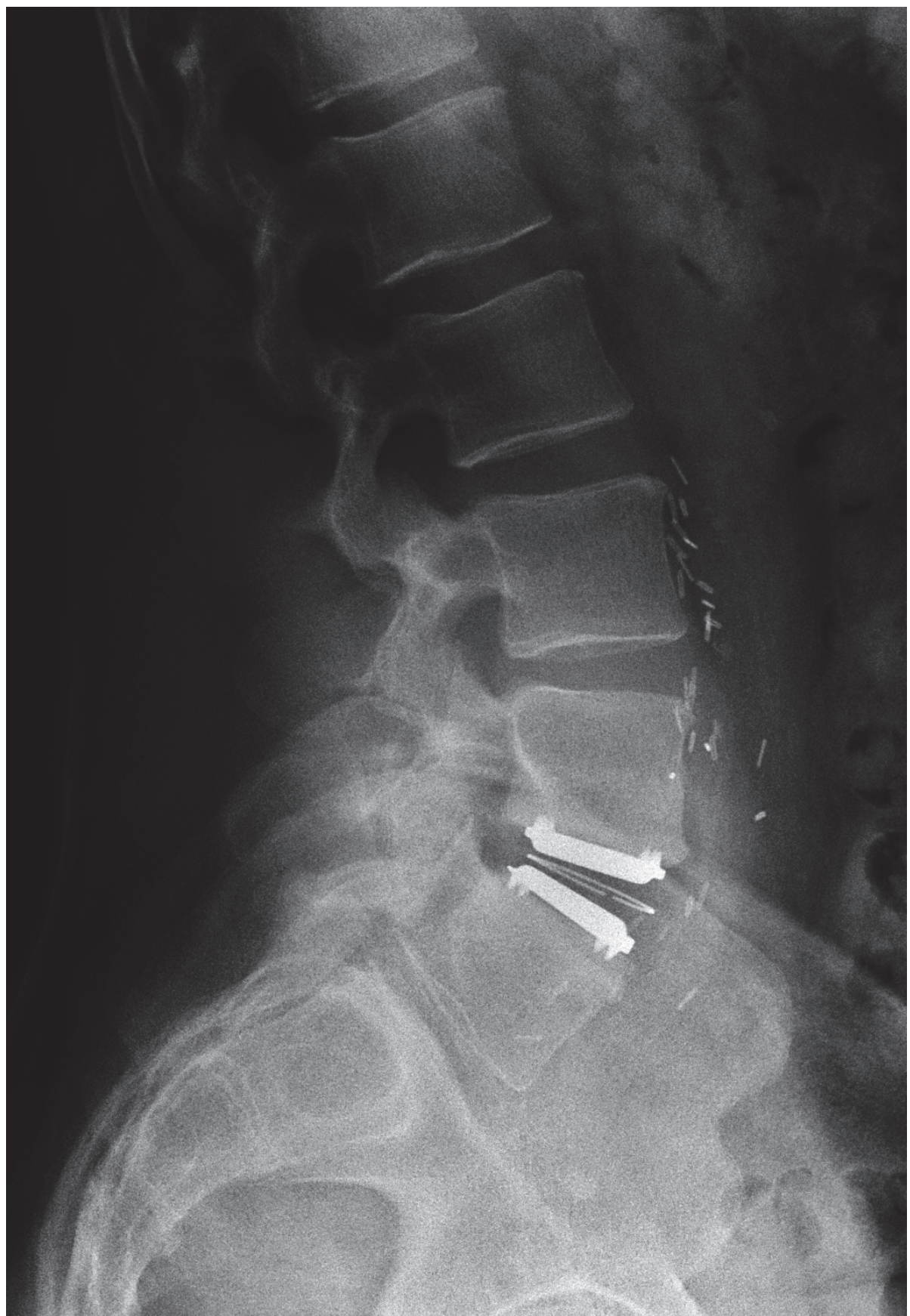

Fig. 1. Typical radiographies for a TDR patient. 1a. Pre-operative MRI. 1b. Post-operative A$P$ radiography. 1c. Post-operative lateral radiography. 


\section{Years: Mean VAS Scores}

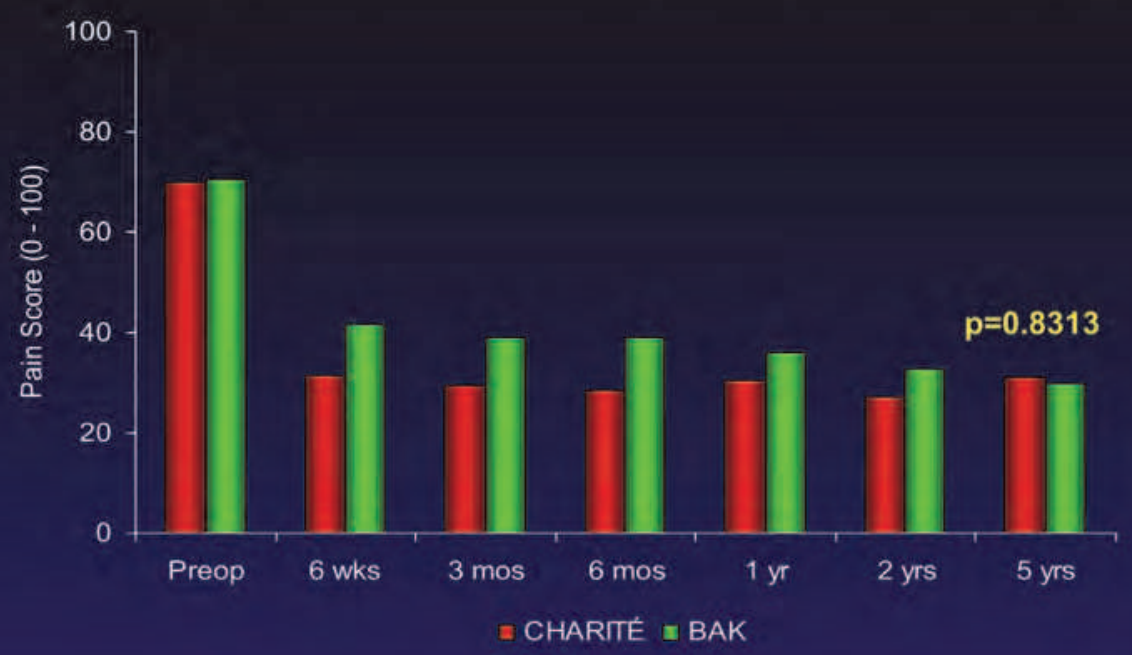

\section{Years: Mean ODI Scores}

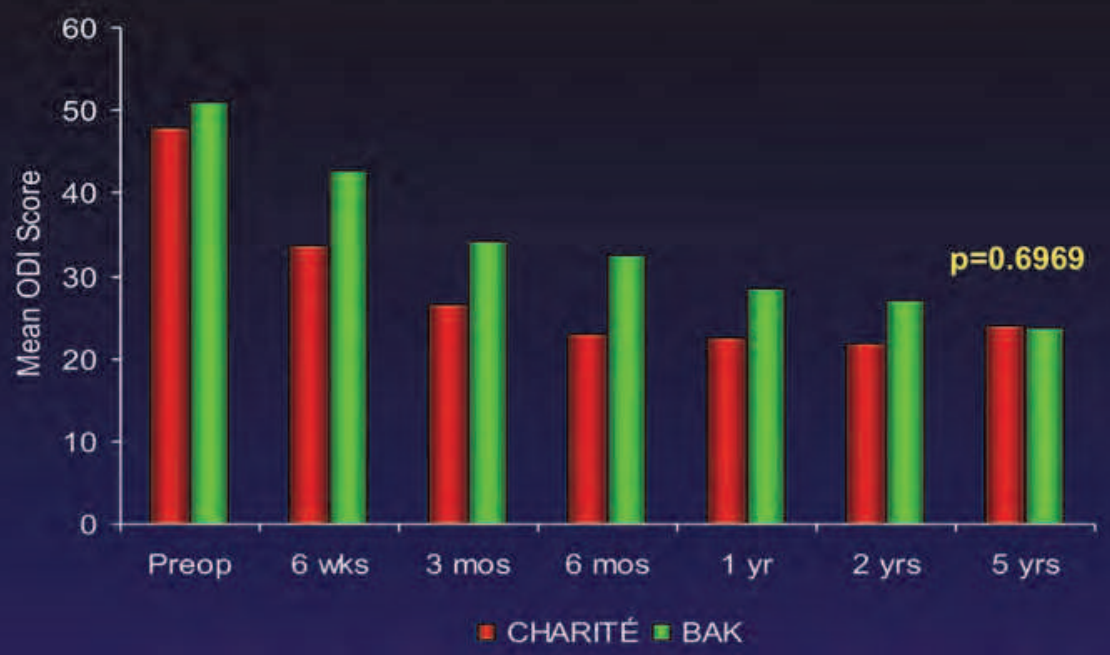

Fig. 2. Five years results from the Charité FDA IDE prospective randomized study. 2a. ODI. 2b. VAS. 


\section{Revision techniques}

If a CHARITÉ artificial disc were required to be revised, there would be two options available. One approach would be anterior reoperation. This would involve dissecting the retroperitoneal area and dealing with the post-op scarring and hence increased risk of great vessel damage, ureteral damage, and damage to the sympathetic nerves compared to a case without prior dissection and scarring in the retroperitoneal space. A revision allows removal, position adjustment or size change of the CHARITÉ artificial disc. The plastic core would be removed first, and then the metal endplates are separated from the bony endplates by using a chisel between them and levering away from the bone into the disc space. This would allow the placement of another artificial disc in the disc space or the conversion to a fusion. Alternately, a posterior operation with rod-screw stabilization and posterior lateral fusion could be used to fuse the lumbar segment, which would use the CHARITÉ artificial disc as an anterior load share device. As with all surgical decision making, understanding the biomechanical reasoning and etiology of clinical failure is of the utmost importance. In patients with recurring or persistent pain the characterization of the pain generator is often more important than the exact surgical technique used. Radiologic studies such as dynamic A-P and Lat x-ray and multislice CT will aid in understanding failure of the device or progression of the degenerative anatomic changes. Radiologic and provocative studies, including discography, anesthetic or negative discography, nerve root blocks, epidural injections, and facet injections may all be utilized to identify the anatomic site of the pain generator.

\section{Long-term follow-up of TDR patients}

The initial CHARITÉ was planned with a 2-year follow-up period. At the request of the FDA the follow-up period was extended to 5 -years and the sites requested to participate in the "new" 2 to 5 year follow-up period. Multiple sites did participate in this extended reporting period and formed the basis of the 5-year CHARITÉ results. The CHARITÉ 5-year ODI and VAS results (Figure $2 \mathrm{a}$ and $2 \mathrm{~b}$ respectively) were substantially the same as the 2-years follow-up results ${ }^{60}$. Despite the prospective collection of the these results, critics formulated objections to these reported good results ${ }^{61}$.

\section{Conclusion}

Overall, there were only minor differences between devices in terms of overall clinical and radiographic outcome. Significant improvements in clinical outcomes were seen with all evaluated devices, regardless of make or design. An average maintenance of motion postoperatively was described, along with relatively low rates of revision. Differences between devices were mostly apparent in complication types: one potential complication for devices with keels was vertebral body split, while devices with metal-on-metal designs could cause metallosis and ion release in the serum. As for metal-on-poly devices, degradation of the polymer core was also mentioned as a potential complication, albeit one that is not relevant for the current metal-on-poly devices. Specific emphasis was found in most of all publications on proper technique and patient selection, regardless of implant design. Finally, arthroplasty was found to be less costly than fusion.

\section{References}

[1] Griffith SL, Shelokov AP, Buttner-Janz K, LeMaire JP, Zeegers WS. A multicenter retrospective study of the clinical results of the LINK SB Charite intervertebral 
prosthesis. The initial European experience. Spine. Aug 15 1994;19(16):18421849.

[2] Zigler J, Delamarter R, Spivak JM, et al. Results of the prospective, randomized, multicenter Food and Drug Administration investigational device exemption study of the ProDisc-L total disc replacement versus circumferential fusion for the treatment of 1-level degenerative disc disease. Spine (Phila $\mathrm{Pa}$ 1976). May 15 2007;32(11):1155-1162; discussion 1163.

[3] McAfee PC, Fedder IL, Saiedy S, Shucosky EM, Cunningham BW. SB Charite disc replacement: report of 60 prospective randomized cases in a US center. J Spinal Disord Tech. Aug 2003;16(4):424-433.

[4] Lemaire JP, Carrier H, Sari Ali E, Skalli W, Lavaste F. Clinical and Radiological Outcomes With the CHARITÉTM Artificial Disc: A 10-Year Minimum Follow-Up. J Spinal Disord. 2005;18(4):353-359.

[5] Guyer RD, McAfee PC, Hochschuler SH, et al. Prospective randomized study of the Charite artificial disc: data from two investigational centers. Spine J. Nov-Dec 2004;4(6 Suppl):252S-259S.

[6] Geisler FH, Blumenthal SL, Guyer RD, et al. Neurological complications of lumbar artificial disc replacement and comparison of clinical results with those related to lumbar arthrodesis in the literature: results of a multicenter, prospective, randomized investigational device exemption study of Charite intervertebral disc. Invited submission from the Joint Section Meeting on Disorders of the Spine and Peripheral Nerves, March 2004. J Neurosurg Spine. Sep 2004;1(2):143-154.

[7] Blumenthal SL, McAfee PC, Guyer RD, et al. A Prospective, Randomized, Multicenter Food and Drug Administration Investigational Device Exemptions Study of Lumbar Total Disc Replacement with the CHARITÉTM Artificial Disc Versus Lumbar Fusion Part I: Evaluation of Clinical Outcomes. Spine. 2005;30(14):15651575.

[8] McAfee PC, Cunningham B, Holsapple GA, et al. A Prospective, Randomized, Multicenter Food and Drug Administration Investigational Device Exemptions Study of Lumbar Total Disc Replacement with the CHARITÉTM Artificial Disc Versus Lumbar Fusion Part II: Evaluation of Radiographic Outcomes and Correlation of Surgical Technique Accuracy with Clinical Outcomes. Spine. 2005;30(14):1576-1583.

[9] David T. Long-term results of one-level lumbar arthroplasty: minimum 10-year followup of the CHARITE artificial disc in 106 patients. Spine (Phila Pa 1976). Mar 15 2007;32(6):661-666.

[10] Ross R, Mirza AH, Norris HE, Khatri M. Survival and clinical outcome of SB Charite III disc replacement for back pain. J Bone Joint Surg Br. Jun 2007;89(6):785-789.

[11] Guyer RD, Blumenthal SL. Survival and clinical outcome of SB Charite III disc replacement for back pain. J Bone Joint Surg Br. Dec 2007;89(12):1673; author reply 1673-1674.

[12] Scott-Young MN. Survival and clinical outcome of SB Charite III disc replacement for back pain. J Bone Joint Surg Br. Dec 2007;89(12):1674; author reply 1674-1675.

[13] Bertagnoli R, Kumar S. Indications for full prosthetic disc arthroplasty: a correlation of clinical outcome against a variety of indications. Eur Spine J. Oct 2002;11 Suppl 2:S131-136. 
[14] Tropiano P, Huang RC, Girardi FP, Cammisa FP, Jr., Marnay T. Lumbar total disc replacement. Seven to eleven-year follow-up. J Bone Joint Surg Am. Mar 2005;87(3):490-496.

[15] Bertagnoli R, Yue JJ, Shah RV, et al. The treatment of disabling single-level lumbar discogenic low back pain with total disc arthroplasty utilizing the Prodisc prosthesis: a prospective study with 2-year minimum follow-up. Spine (Phila $\mathrm{Pa}$ 1976). Oct 1 2005;30(19):2230-2236.

[16] Bertagnoli R, Yue JJ, Shah RV, et al. The treatment of disabling multilevel lumbar discogenic low back pain with total disc arthroplasty utilizing the ProDisc prosthesis: a prospective study with 2-year minimum follow-up. Spine (Phila $\mathrm{Pa}$ 1976). Oct 1 2005;30(19):2192-2199.

[17] Chung SS, Lee CS, Kang CS. Lumbar total disc replacement using ProDisc II: a prospective study with a 2-year minimum follow-up. J Spinal Disord Tech. Aug 2006;19(6):411-415.

[18] Delamarter RB, Fribourg DM, Kanim LE, Bae H. ProDisc artificial total lumbar disc replacement: introduction and early results from the United States clinical trial. Spine (Phila Pa 1976). Oct 15 2003;28(20):S167-175.

[19] Tropiano P, Huang RC, Girardi FP, Marnay T. Lumbar disc replacement: preliminary results with ProDisc II after a minimum follow-up period of 1 year. J Spinal Disord Tech. Aug 2003;16(4):362-368.

[20] Zigler JE, Burd TA, Vialle EN, Sachs BL, Rashbaum RF, Ohnmeiss DD. Lumbar spine arthroplasty: early results using the ProDisc II: a prospective randomized trial of arthroplasty versus fusion. J Spinal Disord Tech. Aug 2003;16(4):352-361.

[21] Zigler JE. Lumbar spine arthroplasty using the ProDisc II. Spine J. Nov-Dec 2004;4(6 Suppl):260S-267S.

[22] Fairbank JC, Pynsent PB. The Oswestry Disability Index. Spine (Phila Pa 1976). Nov 15 2000;25(22):2940-2952; discussion 2952.

[23] Fairbank JC. Use and abuse of Oswestry Disability Index. Spine (Phila Pa 1976). Dec 1 2007;32(25):2787-2789.

[24] Le Huec JC, Mathews H, Basso Y, et al. Clinical results of Maverick lumbar total disc replacement: two-year prospective follow-up. Orthop Clin North Am. Jul 2005;36(3):315-322.

[25] Le Huec JC, Basso Y, Aunoble S, Friesem T, Bruno MB. Influence of facet and posterior muscle degeneration on clinical results of lumbar total disc replacement: two-year follow-up. J Spinal Disord Tech. Jun 2005;18(3):219-223.

[26] Sasso RC, Foulk DM, Hahn M. Prospective, randomized trial of metal-on-metal artificial lumbar disc replacement: initial results for treatment of discogenic pain. Spine (Phila Pa 1976). Jan 15 2008;33(2):123-131.

[27] Cunningham BW, McAfee PC, Geisler FH, et al. Distribution of in vivo and in vitro range of motion following 1-level arthroplasty with the CHARITE artificial disc compared with fusion. J Neurosurg Spine. Jan 2008;8(1):7-12.

[28] Lim MR, Girardi FP, Zhang K, Huang RC, Peterson MG, Cammisa FP, Jr. Measurement of total disc replacement radiographic range of motion: a comparison of two techniques. J Spinal Disord Tech. Jun 2005;18(3):252-256.

[29] Lim MR, Loder RT, Huang RC, et al. Measurement error of lumbar total disc replacement range of motion. Spine (Phila Pa 1976). May 1 2006;31(10):E291-297. 
[30] Huang RC, Girardi FP, Cammisa Jr FP, Tropiano P, Marnay T. Long-term flexionextension range of motion of the prodisc total disc replacement. J Spinal Disord Tech. Oct 2003;16(5):435-440.

[31] Chung SS, Lee CS, Kang CS, Kim SH. The effect of lumbar total disc replacement on the spinopelvic alignment and range of motion of the lumbar spine. J Spinal Disord Tech. Jul 2006;19(5):307-311.

[32] Le Huec J, Basso Y, Mathews H, et al. The effect of single-level, total disc arthroplasty on sagittal balance parameters: a prospective study. Eur Spine J. Jun 2005;14(5):480486.

[33] Tournier C, Aunoble S, Le Huec JC, et al. Total disc arthroplasty: consequences for sagittal balance and lumbar spine movement. Eur Spine J. Mar 2007;16(3):411-421.

[34] McAfee PC, Cunningham BW, Devine J, Williams E, Yu-Yahiro J. Classification of heterotopic ossification $(\mathrm{HO})$ in artificial disk replacement. J Spinal Disord Tech. Aug 2003;16(4):384-389.

[35] Tortolani PJ, Cunningham BW, Eng M, McAfee PC, Holsapple GA, Adams KA. Prevalence of heterotopic ossification following total disc replacement. A prospective, randomized study of two hundred and seventy-six patients. J Bone Joint Surg Am. Jan 2007;89(1):82-88.

[36] Huang RC, Tropiano P, Marnay T, Girardi FP, Lim MR, Cammisa FP, Jr. Range of motion and adjacent level degeneration after lumbar total disc replacement. Spine J. May-Jun 2006;6(3):242-247.

[37] Trouillier H, Kern P, Refior HJ, Muller-Gerbl M. A prospective morphological study of facet joint integrity following intervertebral disc replacement with the CHARITE Artificial Disc. Eur Spine J. Feb 2006;15(2):174-182.

[38] David T. Revision of a Charite artificial disc 9.5 years in vivo to a new Charite artificial disc: case report and explant analysis. Eur Spine J. Jun 2005;14(5):507-511.

[39] Leary SP, Regan JJ, Lanman TH, Wagner WH. Revision and explantation strategies involving the CHARITE lumbar artificial disc replacement. Spine (Phila Pa 1976). Apr 20 2007;32(9):1001-1011.

[40] McAfee PC, Geisler FH, Saiedy S, et al. Revisability of the CHARITÉ Artificial Disc Replacement - Analysis of 688 Patients Enrolled in the U.S. IDE Study of the CHARITÉ Artificial Disc. Spine. 2006 2006;31(11):1217-1226.

[41] Punt IM, Visser VM, van Rhijn LW, et al. Complications and reoperations of the SB Charite lumbar disc prosthesis: experience in 75 patients. Eur Spine J. Jan 2008;17(1):36-43.

[42] van Ooij A, Oner FC, Verbout AJ. Complications of artificial disc replacement: a report of 27 patients with the SB Charite disc. J Spinal Disord Tech. Aug 2003;16(4):369-383.

[43] Geisler FH. Surgical Technique of Lumbar Artificial Disc Replacement with the CHARITETM Artificial Disc. Neurosurgery. 2005;56:ONS46-57.

[44] Gumbs AA, Shah RV, Yue JJ, Sumpio B. The open anterior paramedian retroperitoneal approach for spine procedures. Arch Surg. Apr 2005;140(4):339-343.

[45] Shim CS, Lee S, Maeng DH, Lee SH. Vertical split fracture of the vertebral body following total disc replacement using ProDisc: report of two cases. J Spinal Disord Tech. Oct 2005;18(5):465-469.

[46] Schulte TL, Lerner T, Hackenberg L, Liljenqvist U, Bullmann V. Acquired spondylolysis after implantation of a lumbar ProDisc II prosthesis: case report and review of the literature. Spine (Phila Pa 1976). Oct 15 2007;32(22):E645-648. 
[47] Francois J, Coessens R, Lauweryns P. Early removal of a Maverick disc prosthesis: surgical findings and morphological changes. Acta Orthop Belg. Feb 2007;73(1):122127.

[48] Zeh A, Planert M, Siegert G, Lattke P, Held A, Hein W. Release of cobalt and chromium ions into the serum following implantation of the metal-on-metal Maverick-type artificial lumbar disc (Medtronic Sofamor Danek). Spine (Phila Pa 1976). Feb 1 2007;32(3):348-352.

[49] Bertagnoli R, Yue JJ, Nanieva R, et al. Lumbar total disc arthroplasty in patients older than 60 years of age: a prospective study of the ProDisc prosthesis with 2-year minimum follow-up period. J Neurosurg Spine. Feb 2006;4(2):85-90.

[50] Bertagnoli R, Yue JJ, Kershaw T, et al. Lumbar total disc arthroplasty utilizing the ProDisc prosthesis in smokers versus nonsmokers: a prospective study with 2-year minimum follow-up. Spine (Phila Pa 1976). Apr 20 2006;31(9):992-997.

[51] Bertagnoli R, Yue JJ, Fenk-Mayer A, Eerulkar J, Emerson JW. Treatment of symptomatic adjacent-segment degeneration after lumbar fusion with total disc arthroplasty by using the prodisc prosthesis: a prospective study with 2-year minimum follow up. J Neurosurg Spine. Feb 2006;4(2):91-97.

[52] Hannibal M, Thomas DJ, Low J, Hsu KY, Zucherman J. ProDisc-L total disc replacement: a comparison of 1-level versus 2-level arthroplasty patients with a minimum 2-year follow-up. Spine (Phila Pa 1976). Oct 1 2007;32(21):2322-2326.

[53]Yaszay B, Bendo JA, Goldstein JA, Quirno M, Spivak JM, Errico TJ. Effect of intervertebral disc height on postoperative motion and outcomes after ProDisc-L lumbar disc replacement. Spine (Phila Pa 1976). Mar 1 2008;33(5):508-512; discussion 513.

[54] Guyer RD, Geisler FH, Blumenthal SL, McAfee PC, Mullin BB. Effect of age on clinical and radiographic outcomes and adverse events following 1-level lumbar arthroplasty after a minimum 2-year follow-up. I Neurosurg Spine. Feb 2008;8(2):101-107.

[55] Geisler FH, Guyer RD, Blumenthal SL, et al. Effect of previous surgery on clinical outcome following 1-level lumbar arthroplasty. J Neurosurg Spine. Feb 2008;8(2):108-114.

[56] Geisler FH, Guyer RD, Blumenthal SL, et al. Patient selection for lumbar arthroplasty and arthrodesis: the effect of revision surgery in a controlled, multicenter, randomized study. J Neurosurg Spine. Jan 2008;8(1):13-16.

[57] Guyer RD, Tromanhauser SG, Regan JJ. An economic model of one-level lumbar arthroplasty versus fusion. Spine J. Sep-Oct 2007;7(5):558-562.

[58] Levin DA, Bendo JA, Quirno M, Errico T, Goldstein J, Spivak J. Comparative charge analysis of one- and two-level lumbar total disc arthroplasty versus circumferential lumbar fusion. Spine (Phila Pa 1976). Dec 1 2007;32(25):2905-2909.

[59] Regan JJ, McAfee PC, Blumenthal SL, et al. Evaluation of Surgical Volume and the Early Experience with Lumbar Total Disc Replacement as Part of the IDE Study of the CHARITÉTM Artificial Disc. Spine. Sept 1, 2006 2006;31(19):2270-2276.

[60] Guyer RD, McAfee PC, Banco RJ, et al. Prospective, randomized, multicenter Food and Drug Administration investigational device exemption study of lumbar total disc replacement with the CHARITE artificial disc versus lumbar fusion: five-year follow-up. Spine J. May 2009;9(5):374-386.

[61] van den Eerenbeemt KD, Ostelo RW, van Royen BJ, Peul WC, van Tulder MW. Total disc replacement surgery for symptomatic degenerative lumbar disc disease: a systematic review of the literature. Eur Spine J. Aug 2010;19(8):1262-1280. 


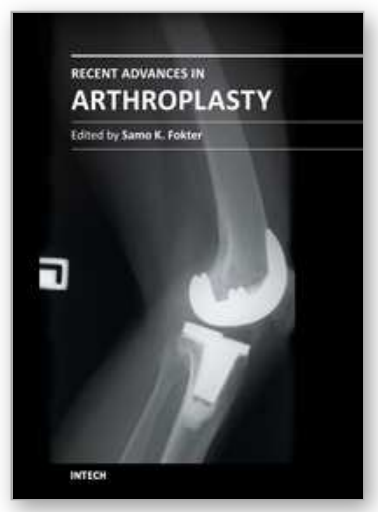

\author{
Recent Advances in Arthroplasty \\ Edited by Dr. Samo Fokter
}

ISBN 978-953-307-990-5

Hard cover, 614 pages

Publisher InTech

Published online 27, January, 2012

Published in print edition January, 2012

The purpose of this book was to offer an overview of recent insights into the current state of arthroplasty. The tremendous long term success of Sir Charnley's total hip arthroplasty has encouraged many researchers to treat pain, improve function and create solutions for higher quality of life. Indeed and as described in a special chapter of this book, arthroplasty is an emerging field in the joints of upper extremity and spine. However, there are inborn complications in any foreign design brought to the human body. First, in the chapter on infections we endeavor to provide a comprehensive, up-to-date analysis and description of the management of this difficult problem. Second, the immune system is faced with a strange material coming in huge amounts of micro-particles from the tribology code. Therefore, great attention to the problem of aseptic loosening has been addressed in special chapters on loosening and on materials currently available for arthroplasty.

\title{
How to reference
}

In order to correctly reference this scholarly work, feel free to copy and paste the following:

Fred H. Geisler (2012). Lumbar Spinal Arthroplasty: Clinical Experience, Recent Advances in Arthroplasty, Dr. Samo Fokter (Ed.), ISBN: 978-953-307-990-5, InTech, Available from:

http://www.intechopen.com/books/recent-advances-in-arthroplasty/lumbar-spinal-arthroplasty-clinicalexperience

\section{INTECH}

open science | open minds

\author{
InTech Europe \\ University Campus STeP Ri \\ Slavka Krautzeka 83/A \\ 51000 Rijeka, Croatia \\ Phone: +385 (51) 770447 \\ Fax: +385 (51) 686166 \\ www.intechopen.com
}

\author{
InTech China \\ Unit 405, Office Block, Hotel Equatorial Shanghai \\ No.65, Yan An Road (West), Shanghai, 200040, China \\ 中国上海市延安西路65号上海国际贵都大饭店办公楼 405 单元 \\ Phone: +86-21-62489820 \\ Fax: +86-21-62489821
}


(C) 2012 The Author(s). Licensee IntechOpen. This is an open access article distributed under the terms of the Creative Commons Attribution 3.0 License, which permits unrestricted use, distribution, and reproduction in any medium, provided the original work is properly cited. 